(C) [2007] IEEE. Reprinted, with permission, from [Xiangjian He, Jianmin Li, Wenjing Jia, Qiang Wu and Tom Hintz,Local Binary Patterns on Hexagonal Image Structure, Computer and Information Technology, 2007. CIT 2007. 7th IEEE International Conference on 16-19 Oct. 2007]. This material is posted here with permission of the IEEE. Such permission of the IEEE does not in any way imply IEEE endorsement of any of the University of Technology, Sydney's products or services. Internal or personal use of this material is permitted. However, permission to reprint/republish this material for advertising or promotional purposes or for creating new collective works for resale or redistribution must be obtained from the IEEE by writing to pubs-permissions@ieee.org. By choosing to view this document, you agree to all provisions of the copyright laws protecting it 


\title{
Local Binary Patterns on Hexagonal Image Structure
}

\author{
Xiangjian $\mathrm{He}^{1}$, Jianmin $\mathrm{Li}^{2}$, Wenjing $\mathrm{Jia}^{1}$, Qiang $\mathrm{Wu}^{1}$ and Tom Hintz ${ }^{1}$ \\ ${ }^{I}$ Department of Computer Systems \\ University of Technology, Sydney \\ Australia \\ \{sean,wejia,wuq,hintz\}@it.uts.edu.au \\ ${ }^{2}$ School of Computer and Mathematics \\ Fuzhou University \\ P.R. China \\ lijianm_9@hotmail.com
}

\begin{abstract}
Local Binary Pattern (LBP) was designed and widely used for efficient texture classification. It has been used for face recognition and has potential applications in many other research areas such as human detection. LBP provides a simple and effective way to represent patterns. Uniform LBPs play an important role for LBP-based pattern lobject recognition as they include majority of LBPs. In this paper, we present $L B P$ codes on hexagonal image structure. We show that LBPs defined on hexagonal structure have higher percentages of uniform LBPs that will lead to a more efficient and accurate recognition scheme for image classification.
\end{abstract}

\section{Introduction}

Local Binary Pattern (LBP), originally designed for efficient texture classification provides a simple and effective way to represent faces [1]. With LBP, face image is divided into small blocks and LBP features are extracted for individual blocks to represent the texture of a face locally and globally. Weighted Chi Square Distance of these LBP histograms is used as a dissimilarity measure for comparing two images. Uniform LBPs as defined in [2] have played an important role for matching based on LBP histograms. Uniform LBPs include most of LBPs and hence all non-uniform LBPs are usually gathered into one single bin of LBP diagrams in order to reduce the number of bins and enhance the classification efficiency. Research works done in [2] and [3] have shown that LBP based methods can produce good results for face recognition in 2D images [1]. Through its recent extensions, the LBP operator has been made into a really powerful measure of image texture, showing excellent results in terms of accuracy and computational complexity in many empirical studies. The LBP operator can be seen as a unifying approach to the traditionally divergent statistical and structural models of texture analysis.

In this paper, we construct LBP based on a hexagonal image structure [4]. We will use experimental results to show that LBPs defined on the hexagonal structure have much higher percentages of uniform LBPs. Therefore, LBP-based object/pattern recognition on hexagonal structure has potentials for more accurate and efficient classification.

In order to show another potential application of LBPs, LBP maps obtained from Mahalanobis distance maps [5] will be examined on both square structure and hexagonal structure for human detection.

The rest of this paper is organized as follows. In Section 2, a hexagonal structure is briefly introduced. We review LBP coding methods on traditional square structure in Section 3. LBP coding on hexagonal structure is presented in Section 4. Experimental results that compare LBPs on square and hexagonal structures are demonstrated in Section 5. We conclude in Section 6.

\section{Hexagonal Image Structure}

The arrangement of a hexagonal grid is different from a rectangular grid as seen in Figure 1. The advantages of using a hexagonal grid to represent digit images have been investigated for more than thirty years. The importance of the hexagonal representation 
is that it possesses special computational features that are pertinent to the vision process [6]. The hexagonal image structure has features of higher degree of circular symmetry, uniform connectivity, greater angular resolution, and a reduced need of storage and computation in image processing operations [4].

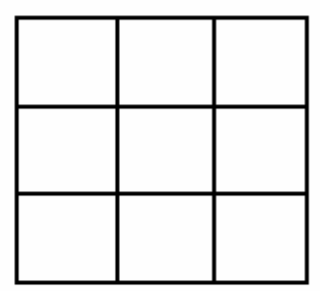

(a) Rectangular

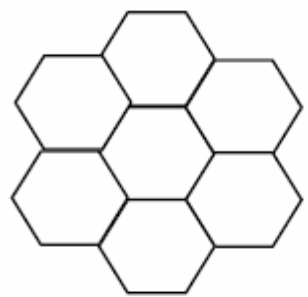

(b) Hexagonal Architecture

\section{Architecture}

\section{Figure 1. Vision unit in two different image architectures}

\subsection{Construction of a Virtual Hexagonal Structure}

Because there has been no hardware available for image display and capture on hexagonal structure, in this section, we review a software approach to the construction of virtual hexagonal structure [7, 8]. To construct hexagonal pixels, in [7], each square pixel was first separated into $7 \times 7$ small pixels, called $s u b$ pixels. Each virtual hexagonal pixel was formed by 56 sub-pixels as shown in Figure 2. Figure 2 shows a collection of seven hexagonal pixels. The light intensity of any given virtual hexagonal pixel is assigned using the light intensity of one of its 56 subpixels that is located at the forth row and the middle column of these 56 sub-pixels. This sub-pixel is called the reference sub-pixel of the corresponding virtual hexagonal pixel close to the centre of the hexagonal pixel like the sub-pixels $\mathrm{P}_{\mathrm{i}}(\mathrm{i}=0,1,2, \ldots, 6)$ shown in Figure 3 . The light intensity of each virtual hexagonal pixel is approximated through bi-linear interpolation process as shown in [8].

It is not difficult to locate each virtual hexagonal pixel when the size of an image is known [7].

\subsection{Conversion from Virtual Hexagonal Structure to Square Structure}

Converting images from the virtual structure to the square structure can be simply performed in a similar way as from square to hexagonal. The light intensity of any given square pixel is assigned using the light intensity of the sub-pixel that is located at the centre of these $7 \times 7$ sub-pixels that were separated from the square pixel. The light intensities of these central subpixels are re-assigned through a tri-linear interpolation process as shown in [8].

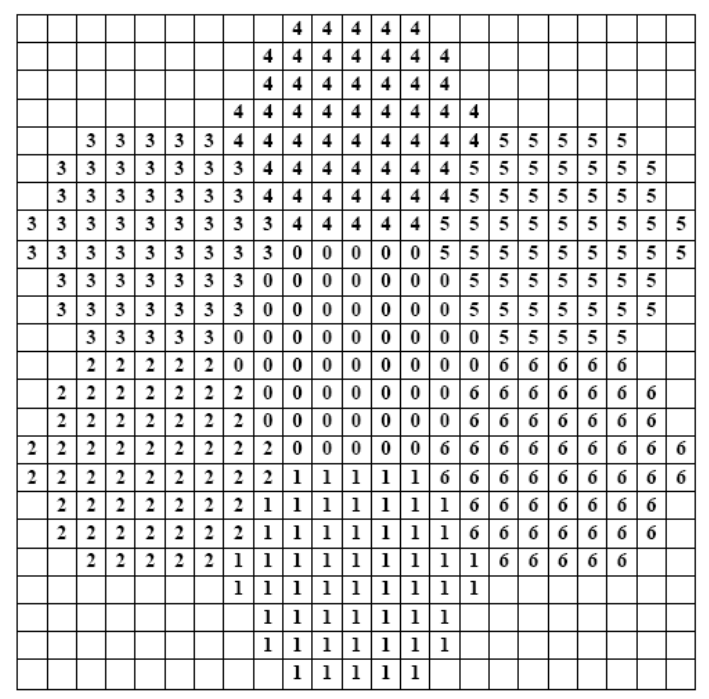

Figure 2. A cluster of seven hexagonal pixels

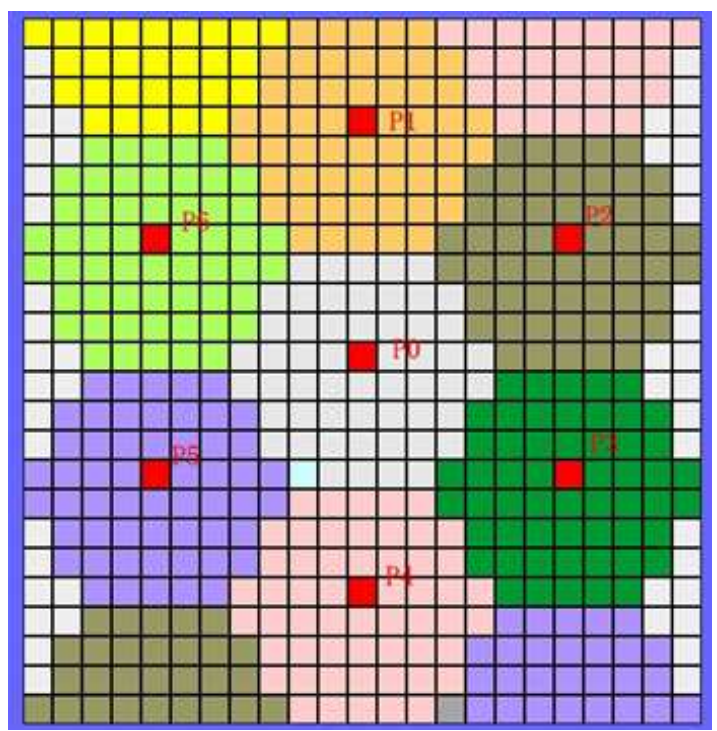

Figure 3. Reference sub-pixels of virtual hexagonal pixels 


\section{LBP on Square Structure}

LBP was originally introduced by Ojala et al. in [9] as texture description. LBP features have performed very well in various applications including texture classification and segmentation [1]. The basic form of an LBP operator on square image structure labels the pixels of an image by thresholding the $3 \times 3$ neighborhood of each pixel by the grey value of the pixel (the centre). An illustration of the basic LBP operator is shown in Figure 4. Note that the binary LBP code is circular. The basic LBP consists of 8 binary bits representing an integer from 0 to 255 .

The major limitation of the basic LBP operator is that the 8 neighboring pixels are not exactly on a circle because of the unequal distances between the reference pixel and its neighbors. Grey values found in the 8 neighbors may not be equally dominant to the reference pixel.

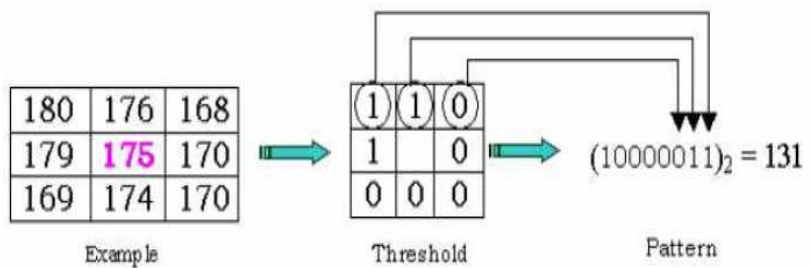

Figure 4. Calculation of LBP code from $3 \times 3$ neighborhood [3]

In order to reduce the complexity of computation, uniform LBPs have been defined as shown in [1]. A uniform LBP is a LBP that contains at most two bit wise $0-1$ or 1-0 transitions [1]. For example, 00000000 , 00001000,11000111 are uniform patterns. With this extension, there are 58 uniform LBP code patterns for 8 bit basic LBP code, and 256-58=198 non-uniform LBP code patterns. These uniform LBP codes function as templates for microstructure such as bright spot (00000000), flat area or dark spot (11111111), and edges of varying positive and negative curvatures (others) [10].

\section{LBP on Hexagonal Structure}

Similar to the construction of basic LBP on square structure, we now construct basic LBP on hexagonal structure as shown in Figure 5.

By defining the basic LBP codes on hexagonal structure, the number of different patterns has been reduced from $2^{8}=256$ on square to $2^{6}=64$ on hexagonal structure. More importantly, because all neighboring pixels of a reference pixel have the same distance to it, the grey values of the neighboring pixels have the same contributions to the reference pixel on hexagonal. Furthermore, because all six neighboring pixels are exactly on a circle with radius 1 (assuming the distance between neighboring hexagonal pixels is 1) and centre at the reference pixel, unlike on square structure, there is no longer need to perform an interpolation process before computing the LBP codes on hexagonal structure.

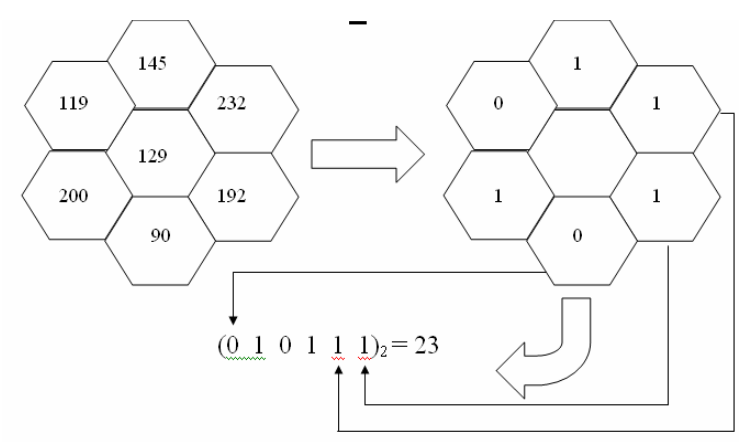

Figure 5. Calculation of basic LBP code on hexagonal structure

\subsection{Uniform LBPs}

If we define a uniform LBP on hexagonal structure in the same way as on square such that each uniform LBP contains only two 1-0 or 0-1 transitions, then the number of uniform LBP codes is reduced from 58 on square structure to 32 on hexagonal structure. Like uniform LBPs on square structure, uniform LBPs on hexagonal structure function as bright spot (000000), flat area or dark spot (111111), and edges of varying positive and negative curvatures (others).

Because of smaller number of uniform LBPs compared with those on square structure and more even contribution of neighboring pixels to their reference pixel, one can expect that image recognition based on hexagonal structure using LBPs not only improves the recognition accuracy but also increases the recognition speed.

\subsection{LBP Histograms}

As shown in [10] that uniform patterns accounted about $90 \%$ of all patterns in the experiments with texture images on square structure, it is expected that this percentage will be a lot higher on hexagonal structure because the proportion of uniform LBPs to total LBPs (which is $1 / 2$ ) is more than twice larger on hexagonal than on square structure (which is 29/128). 
Like the work done in $[1,2]$, we consider only the 32 uniform LBP code patterns as 32 LBP types plus one single type that consists of all non-uniform LBP codes. For each pixel $p$, let $L B P(p)$ denote the $L B P$ type at $p$. Then,

$$
\operatorname{LBP}(p) \in L, L=\{0,1, \cdots, 32\},
$$

for given $p$. Let $S$ denote the image plane, and $H(i)$ ( $i=$ $0,1, \ldots, 32$ ) denote the LBP histogram over $S$ corresponding to bin value $i$, i.e.,

$$
H(i)=\sum_{p \in S} I\{L B P(p)=i\},
$$

where

$$
I\{A\}= \begin{cases}1, & A=\text { true }, \\ 0, & A \neq \text { true } .\end{cases}
$$

We can now define the LBP histogram $H$ over $S$ by

$$
H=(H(0), H(1), \cdots, H(32)) .
$$

The histogram $H$ contains information about the distribution of the micro-patterns, such as edges, spots and flat areas, over the image plane $S$.

\section{Experimental Results}

To compare LBPs on square structure and hexagonal structure, 8-bit grey level Lena image (see Figure 6) of size $254 \times 254$ initially represented on square structure is chosen first as a sample image to be processed.

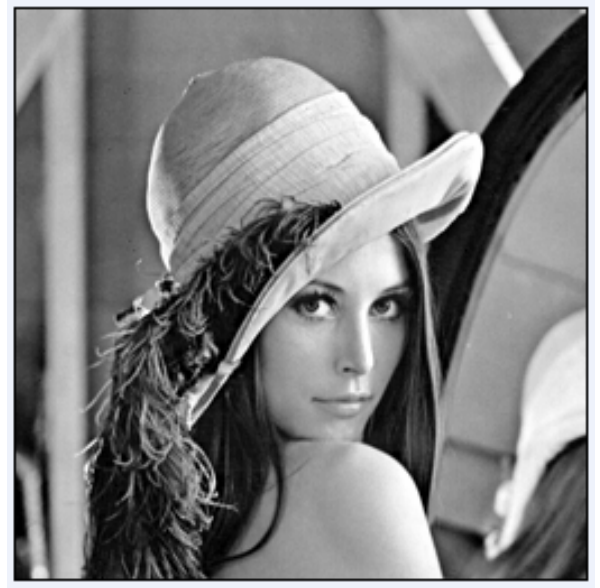

Figure 6. Original Lena image
Figure 7 shows two LBP maps. The top image is obtained on square structure and the bottom one on the virtual hexagonal structure as described in Section 2. The bottom image looks darker because the range of LBP values is smaller and is from 0 to 63. It is easy to see that the bottom image shows clearer edge boundaries than the top image (see Figure 8 for edge images obtained from Figure 7). This can be seen at the boundary of the hat.
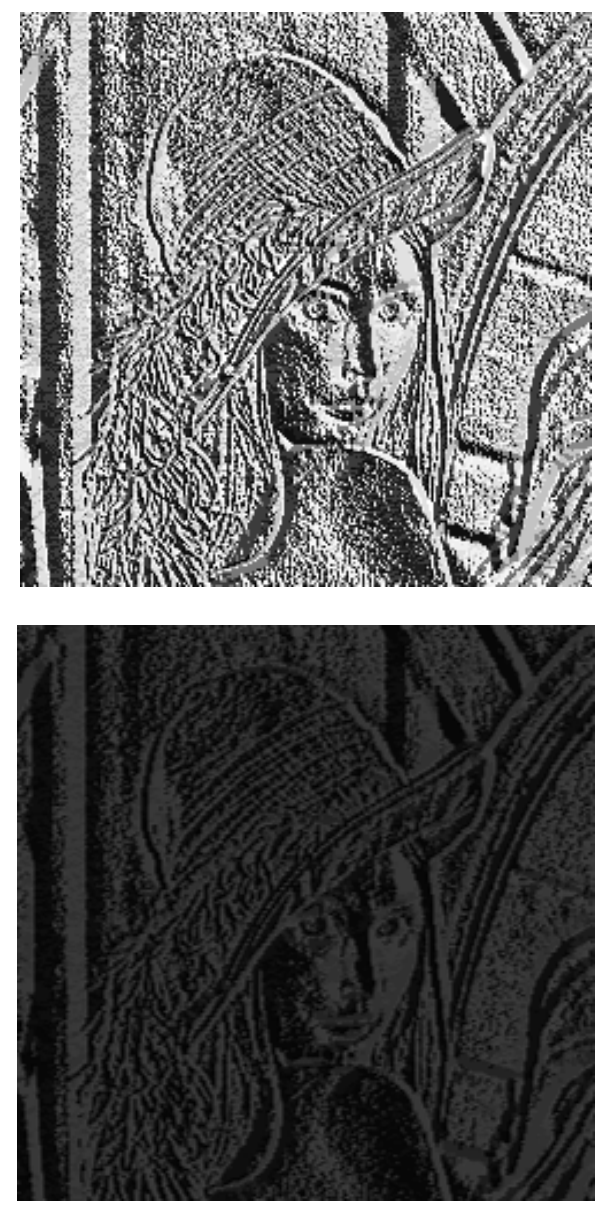

Figure 7. LBP images. Top: on square structure; bottom: on virtual hexagonal structure

It is also found that, in Figure 7, the bottom image contains less non-uniform LBPs because it has less pixels which are neither bright spots, dark spots nor edges. This finding is confirmed by our experimental results showing that the top LBP image contains 13,868 non-uniform LBPs while bottom LBP image contains only 5,037 non-uniform LBPs. This indicates that the histogram of uniform LBPs on hexagonal 
structure includes more pixels while having less bins (see Figure 9). Therefore, LBP defined on hexagonal can give more accurate and also more efficient algorithms for object/pattern recognition and texture classification.
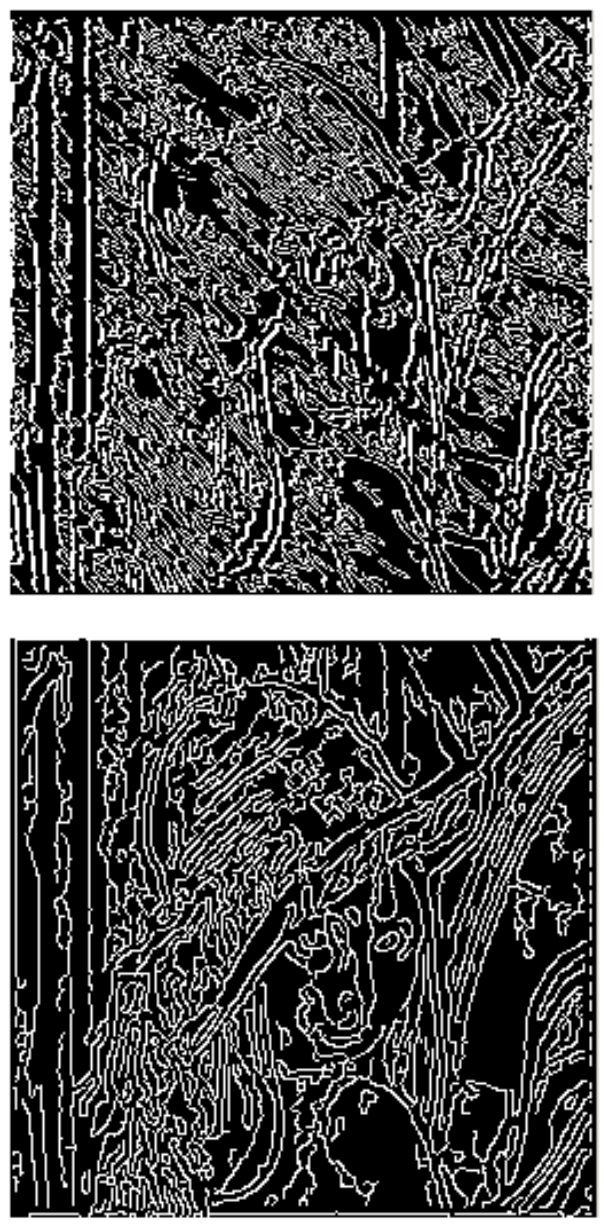

Figure 8. Canny edge maps of images shown in Figure 7. Top: on square structure; bottom: on virtual hexagonal structure

In order to be more promising and to show another potential application, we have also compared LBP histograms on square structure and the virtual structure for human detection. Three Mahalanobis distance maps extracted from [5] are used and displayed in Figure 10. The numbers of uniform LBPs and non-uniform LBPs on both square structure and the virtual hexagonal structure are listed in Table 1. It is again shown that there are only about half non-uniform LBPs on the virtual hexagonal structure than on square structure. It is also shown that there are only small amounts of non- uniform LBPs compared to the amounts of uniform LBPs.
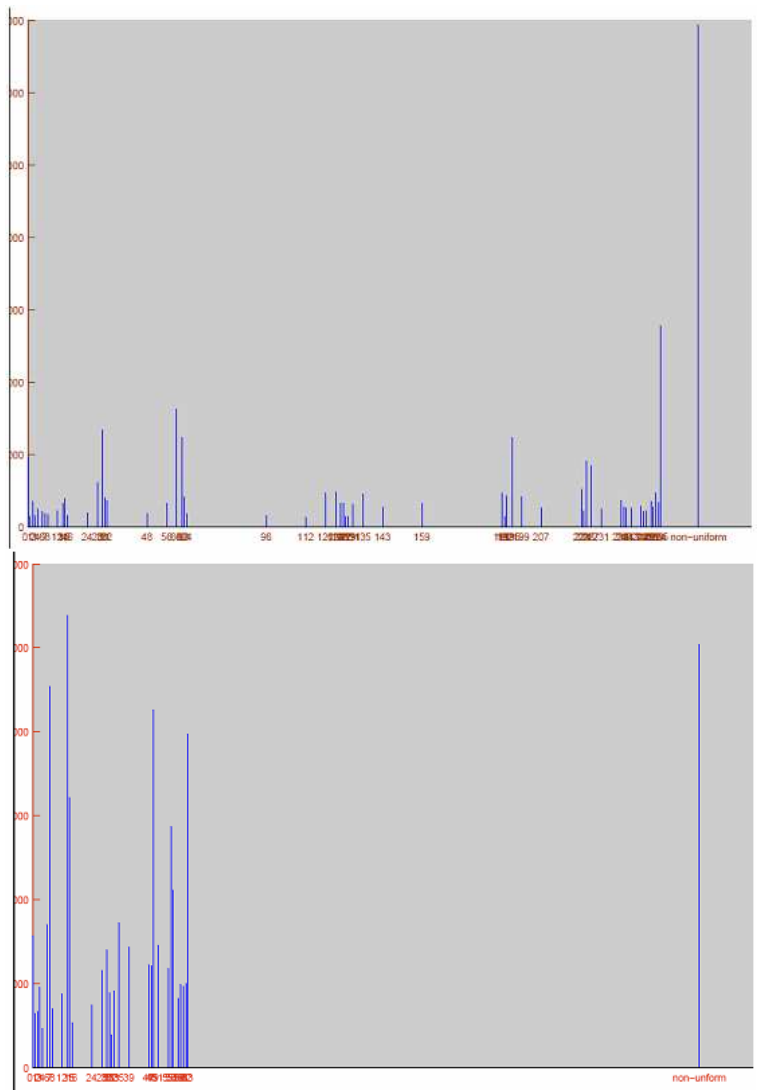

Figure 9. LBP histograms obtained from Figure 7. Top: on square structure; bottom: on virtual hexagonal structure.

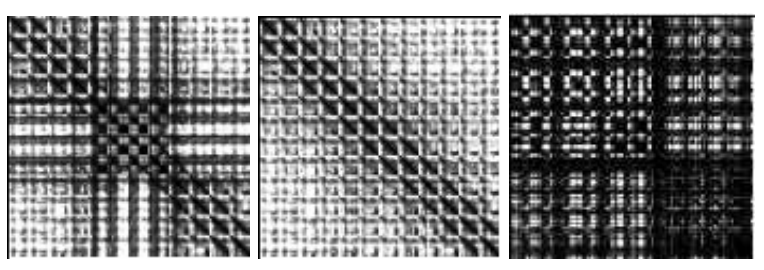

Figure 10. Mahalanobis distance maps. Left: model human map; middle: mode non-human map; right: candidate human map

Table 1. LBP numbers of maps in Figure 10

\begin{tabular}{|l|l|l|l|}
\hline & Human Model & Non-human model & Human candidate \\
\hline Uniform LBP-SQ & 10115 & 10080 & 8901 \\
\hline Non-uniform LBP-SQ & 2429 & 2464 & 3643 \\
\hline Uniform LBP-Hex & 9889 & 9886 & 9392 \\
\hline Non-uniform LBP-Hex & 1298 & 1301 & 1794 \\
\hline
\end{tabular}


When Chi-square measure as shown in [2] is applied to the LBP histograms, it shows that on both square structure and hexagonal structure the human candidate matches the human model better than the non-human model. More results about LBP-based human detection will be discussed in another paper.

\section{Conclusions}

In this paper, we have presented LBPs on hexagonal structure for pattern/object recognition. Compared to LBPs defined on square structure, LBPs on hexagonal structure have potential for more accurate description of texture. Because the number of LBP types and their uniform subset have been greatly reduced on hexagonal structure than on square structure, object/pattern recognition using LBPs on hexagonal structure is much more efficient.

In order to show the potential applications of LBPs on hexagonal structure, LBPs obtained from Mahalanobis distance maps for human detection has also been shown. Encouraging and promising results for human detection have been achieved.

\section{References}

[1] Stan Z. Li, ChunShui Zhao, XiangXin Zhu, Zhen Lei, 2D+3D Face Recognition by Fusion at Both Feature and Decision Levels, In Proceedings of IEEE International Workshop on Analysis and Modeling of Faces and Gestures. Beijing. Oct 16, 2005.

[2] T. Ahonen, A. Hadid and M. Pietikainen, Face recognition with local binary patterns, ECCV2004, Prague, Czech, 2004, pp.469-481.

[3] Hadid, M. Pietikainen and T. Ahonen, A discriminative feature space for detecting and recognizing faces, CVPR2004, pp.797-804.
[4] Xiangjian He, Wenjing Jia, Namho Hur, Qiang $\mathrm{Wu}$, Jinwoong Kim and Tom Hintz, Bi-lateral Edge Detection on a Virtual Hexagonal Structure, Lecture Notes in Computer Science, LNCS, Springer, 2006, Vol.4292, pp.1092-1101.

[5] A. Utsumi and N. Tetsutani, Human detection using geometrical pixel value structure, $5^{\text {th }}$ IEEE International Conference on Automatic Face and Gesture Recognition, FGR2002.

[6] Qiang Wu, Xiangjian He, Tom Hintz and Yuhuang Ye, A Novel and Uniform Image Separation on Spiral Architecture, International Journal of Computational Science and Engineering, Inderscience, Vol.2, Nos.1/2, pp.57-63, 2006.

[7] Xiangjian He, Huaqing Wang, Namho Hur, Wenjing Jia, Qiang Wu, Jinwoong Kim, and Tom Hintz, Uniformly Partitioning Images on Virtual Hexagonal Structure, 9th International Conference on Control, Automation, Robotics and Vision (IEEE ICARCV06), 2006, pp. pp.891-896.

[8] Xiangjian He, Jianmin $\mathrm{Li}$ and Tom Hintz, Comparison of Image Conversions between Square Structure and Hexagonal Structure, Lecture Notes in Computer Science (ACIVS2007), to appear.

[9] T. Ojala, M. Pietikainen and D. Harwood, A comparative study of texture measures with classification based on feature distributions, Pattern Recognition, Vol.29, 2996, pp.51-59.

[10] T. Ojala and M. Pietikainen, Multiresolution gray-scale and rotation invariant texture classification with local binary patterns, IEEE Transactions on Pattern Analysis and Machine Intelligence, Vol.24, No7, 2002, pp.971-987. 